\title{
Persistent pruritic papules and plaques: a characteristic histopathologic presentation seen in a subset of patients with adult-onset and juvenile Still's disease
}

\begin{abstract}
Background: 'Persistent pruritic papules and plaques' of Still's disease represents a recently described eruption seen in a subset of patients. Most cases reported in the literature to date have been associated with adult-onset Still's disease.

Methods: We present the clinical and histopathologic examinations of three female patients ranging in age from 15 to 54 years.

Results: Our three patients each presented with clinical findings consistent with Still's disease. The youngest patient suffered from the juvenile form of Still's disease (systemic-onset juvenile rheumatoid arthritis). Each patient had a persistent, pruritic eruption with histopathologic findings of dyskeratosis confined to the upper layers of the epidermis as well as a sparse superficial dermal infiltrate containing scattered neutrophils.
\end{abstract}

Conclusions: These cases confirm the characteristic clinical and histopathologic findings of 'persistent papules and plaques of Still's disease' and show the potential for this eruption in both the adult and juvenile age groups.

Fortna RR, Gudjonsson JE, Seidel G, DiCostanzo D, Jacobson M, Kopelman M, Patel RM. Persistent pruritic papules and plaques: a characteristic histopathologic presentation seen in a subset of patients with adult-onset and juvenile Still's disease.

J Cutan Pathol 2010; 37: 932-937. (C) 2010 John Wiley \& Sons A/S.
Ryan R. Fortna ${ }^{1}$, Johann E. Gudjonsson ${ }^{2}$, Gregory Seidel ${ }^{3,4}$, Damian DiCostanzo ${ }^{3,4}$, Mark Jacobson ${ }^{3,4}$, Margaret Kopelman' ${ }^{2}$ and Rajiv M. Patel ${ }^{1,2}$

\section{${ }^{1}$ Department of Pathology, \\ 2Department of Dermatology, University of Michigan Medical Center, Ann Arbor MI 48109, USA \\ ${ }^{3}$ Department of Pathology, Montefiore Medical Center, Albert Einstein College of Medicine, Bronx, NY 10461, USA and ${ }^{4}$ Dermpath Diagnostics, Port Chester, NY 10573}

\section{Rajiv M. Patel,}

Departments of Pathology and Dermatology,

University of Michigan Medical Center,

M3261 Medical Sciences I,

1301 Catherine Rd.,

Ann Arbor, MI 48109, USA

Tel: +734 7644460

Fax: +7347644690

e-mail: rajivpat@med.umich.edu,

pate0057@gmail.com

Accepted for publication April 19, 2010
Still's disease is an idiopathic inflammatory disorder with varied clinical manifestations. Its diagnosis is one of exclusion and is often difficult and delayed. The four cardinal features of the disorder include polyarthritis/polyarthralgia, high spiking fevers, leukocytosis with neutrophilia, and evanescent skin rash. Whether the disease presents in adulthood [adult-onset Still's disease (AOSD)] or childhood [systemic form of juvenile idiopathic arthritis (systemic JIA)], skin manifestations are often a critical component of disease recognition. The classic Still's rash consists of an evanescent, non-pruritic, salmon-colored morbilliform eruption; however, a distinct type of Still's rash has recently been described, which consists of persistent (fixed), pruritic papules and plaques which often contain fine scale. ${ }^{1-5}$ The clinicopathologic features of the latter eruption appear to be sufficiently characteristic as to be useful in aiding the diagnosis of the disorder. We present three patients with a clinical diagnosis of 


\section{Persistent pruritic papules and plaques in Still's disease}

Still's disease and persistent rashes that clinically and histopathologically fit the newly described 'persistent pruritic papules and plaques' of Still's disease.

\section{Results}

Case 1

\section{Clinical findings}

Patient 1 was a 15 -year-old fair-skinned female who initially presented with fevers, fatigue, a sore throat and a painful rash of her genitalia, which was subsequently diagnosed as herpes zoster by viral cultures and successfully treated with antiviral therapy. After complete resolution of the zoster, she continued to have fatigue and worsening fevers; subsequently, she experienced polyarthralgia, generalized myalgia, high fevers $\left(39.7^{\circ} \mathrm{C}\right)$ and neurologic symptoms (an episode of ptosis and optical blurriness). Laboratory studies revealed a leukocytosis $\left(15,600\right.$ cells $\left./ \mathrm{mm}^{3}\right)$ with $85 \%$ neutrophils, elevated erythrocyte sedimentation rate $(92 \mathrm{~mm} / \mathrm{h})$, elevated C-reactive protein $(7.4 \mathrm{mg} / \mathrm{dl})$, and markedly elevated serum ferritin $(>16,500 \mathrm{ng} / \mathrm{ml})$. A diagnosis of JIA was made after an extensive infectious, neoplastic and rheumatologic workup was otherwise negative.

The patient began to experience pruritus of the mid-lower back approximately two months after the onset of systemic symptoms. Skin examination at the time of dermatologic consultation revealed fixed, pruritic, mildly scaly, pink to erythematous, blanchable plaques on the lower back, posterior neck/upper back and upper abdomen (Fig. 1A,B). Treatment consisting of anakinra, methotrexate and methylprednisolone resulted in resolution of the cutaneous eruption and a significant improvement in the arthralgias and systemic symptoms.

\section{Histopathologic findings}

Punch biopsies were obtained from the lower back and upper abdomen. Histopathologic examination showed similar findings (Fig. 1G-F), which included hyperkeratosis with patchy parakeratosis, multiple areas of dyskeratosis confined to the upper layers of the epidermis, mild acanthosis, and a sparse superficial and mid-dermal mixed infiltrate consisting of lymphocytes admixed with neutrophils and occasional eosinophils. There was no significant dermal mucin deposition.

\section{Case 2}

\section{Clinical findings}

Patient 2 was a 54-year-old Asian female who developed a sore throat with a rash and high fevers $\left(39.0^{\circ} \mathrm{C}\right)$ preceded by polyarthralgia of the ankles, hips and shoulders. The rash consisted of fixed, pruritic papules and plaques with blanchable erythema and fine scale involving the proximal extremities, back, abdomen and ankles. Laboratory evaluation revealed elevated liver enzymes (ALT $451 \mathrm{IU} / 1$, AST $491 \mathrm{IU} / \mathrm{l}$ ), elevated erythrocyte sedimentation rate $(44 \mathrm{~mm} / \mathrm{h})$, elevated C-reactive protein $(2.0 \mathrm{mg} / \mathrm{dl})$, a leukocytosis of 16,600 cells $/ \mathrm{mm}^{3}$ with $85 \%$ neutrophils, and markedly elevated serum ferritin $(>16,500 \mathrm{ng} / \mathrm{ml})$. An extensive rheumatologic and infectious workup was otherwise negative, and a diagnosis of AOSD was made. The patient was treated with prednisone and later with methylprednisolone, and her symptoms eventually resolved approximately 1.5 months after onset.

\section{Histopathologic findings}

A punch biopsy from the lower back was obtained. Histopathologic assessment revealed predominantly orthokeratotic hyperkeratosis and dyskeratosis confined to the upper portion of the epidermis (Fig. 2A-C). Subtle vacuolar interface alteration was present, but there was little or no inflammatory infiltrate along the dermal-epidermal junction and no evidence of basilar dyskeratosis (Fig. 2B,C). The superficial dermis contained a mild perivascular inflammatory infiltrate with occasional neutrophils and rare eosinophils (Fig. 2D). There was no significant dermal mucin deposition.

\section{Case 3}

\section{Clinical findings}

Patient 3 was a 53-year-old fair-skinned female who had initially been diagnosed with AOSD several years before presentation to the dermatology clinic. She presented with a disease flare that included spiking fevers, polyarthralgia, a leukocytosis $\left(>18,000\right.$ cells $\left./ \mathrm{mm}^{3}\right)$, and markedly elevated ferritin $(14,668 \mathrm{mg} / \mathrm{ml})$. In addition, she exhibited both the classic transient morbilliform Still's rash and persistent, erythematous, slightly scaly papules with somewhat linear configuration on the upper back and chest (Fig. 3A,B). The patient died shortly after this presentation from unrelated causes.

\section{Histopathologic findings}

Two biopsies of the persistent papules from the back showed similar histopathologic findings (Fig. 3C,D). The epidermis was acanthotic with dyskeratotic keratinocytes present predominantly within the upper portion of the epidermis and within a hyperkeratotic stratum corneum. There was 


\section{Fortna et al.}
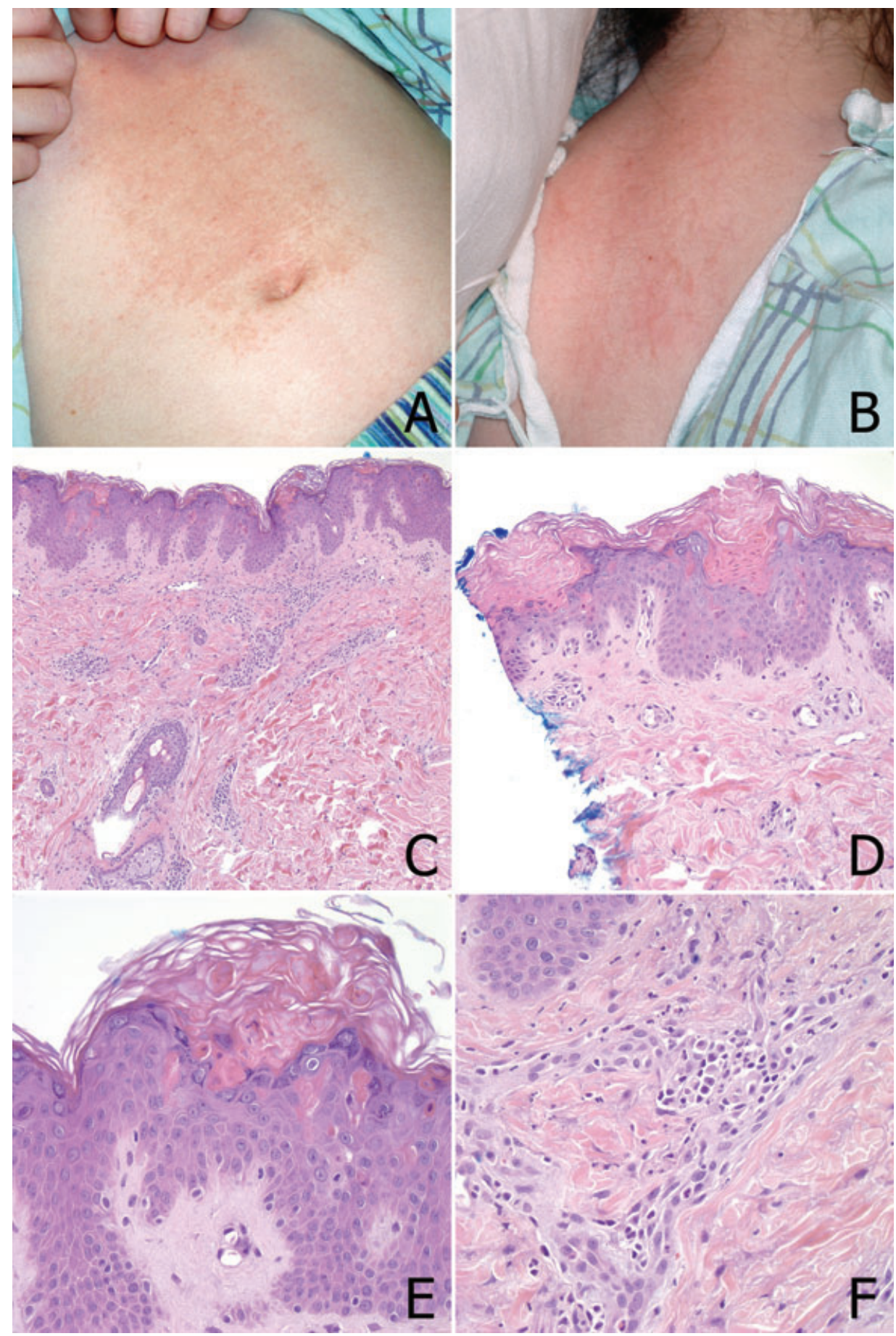

Fig. 1. Clinical and biopsy findings from patient 1. Fixed, pruritic, mildly scaly, pink to erythematous, blanchable plaques were present on the upper abdomen (A), posterior neck/upper back (B), and lower back. Low $(\mathrm{C}, \times 10)$, medium $(\mathrm{D}, \times 20)$ and high $(\mathrm{E}, \mathrm{F}, \times 40)$ power images of the biopsy display patchy dyskeratosis confined to the upper layers of the epidermis, hyperkeratosis with foci of parakeratosis, and a superficial and mid-dermal perivascular and interstitial infiltrate comprised predominantly of neutrophils and lymphocytes with occasional karyorrhectic debris.

a mild superficial dermal perivascular inflammatory infiltrate composed of lymphocytes and scattered neutrophils, and there was no apparent interface reaction. Mild mucin deposition was apparent in the dermis.

\section{Discussion}

Still's disease is an idiopathothic seronegative (rheumatoid factor and antinuclear antibody negative) arthritis. ${ }^{6-8}$ It is divided into juvenile and adult forms, with patients 16 years of age and older 
Persistent pruritic papules and plaques in Still's disease

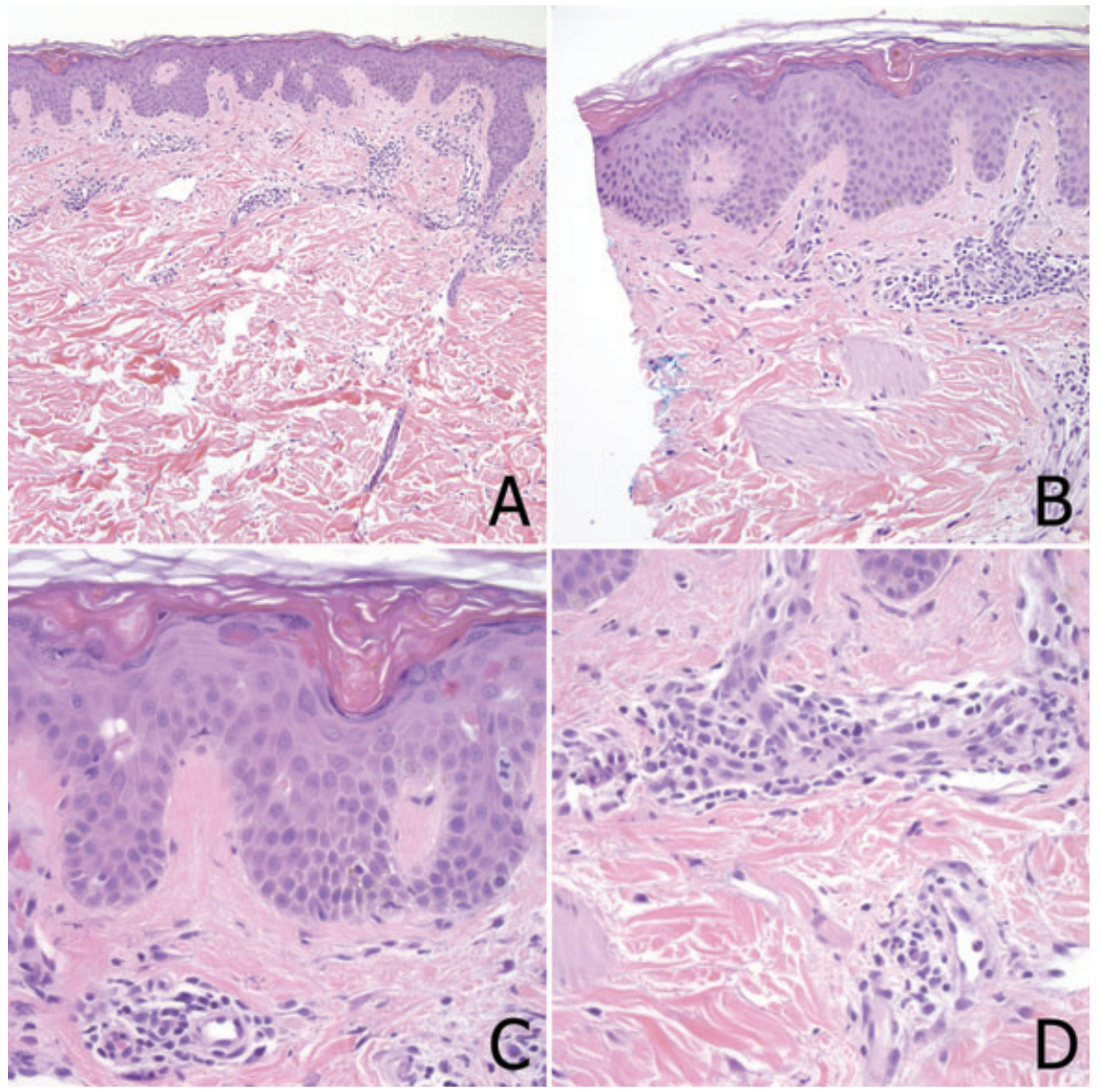

Fig. 2. Biopsy findings from patient 2. Low $(A, \times 10)$, medium $(B, \times 20)$, and high $(\mathrm{C}, \mathrm{D}, \times 40)$ power images of the biopsy display patchy upper-layer dyskeratosis, mild hyperkeratosis, and a mild superficial dermal infiltrate comprised of lymphocytes with occasional neutrophils and rare eosinophils. Occasional foci suggestive of mild vacuolar interface alteration were seen (B, right side), although there was no associated basilar dyskeratosis and little or no inflammation at the dermal-epidermal junction.

classified as adults and the remainder classified as juveniles. The juvenile form corresponds to the systemic subtype of JIA (also known as juvenile rheumatoid arthritis). The myriad signs and symptoms of the disease are generally similar between adult and juvenile subtypes. The four cardinal symptoms of Still's disease include arthritis/arthralgias, high spiking fevers $\left(\geq 39^{\circ} \mathrm{C}\right)$, evanescent rash, and leukocytosis $\geq 10,000$ cells $/ \mathrm{mm}^{3}$ with $\geq 80 \%$ neutrophils. ${ }^{6-8}$ Multiple other signs and symptoms, including sore throat, lymphadenopathy, splenomegaly and hepatic dysfunction may also be present. Laboratory studies typically reveal a markedly elevated serum ferritin level, which is characteristic but not absolutely specific for the disease. The specific diagnostic criteria and differential diagnostic considerations for both adult and juvenile Still's disease have been reviewed previously. ${ }^{6-8}$

The classic rash of Still's disease consists of an evanescent, salmon-colored, morbilliform eruption on the trunk and/or extremities, which typically is asymptomatic but may occasionally be pruritic. ${ }^{9}$ The histopathology of the evanescent rash typically consists of a sparse perivascular mixed inflammatory infiltrate including neutrophils, without epidermal changes. More recently, an eruption has been reported in Still's patients with clinical and histopathologic features clearly distinct from the classic evanescent rash. The first clear description of this specific eruption was presented by Lủbbe et al. in 1999. ${ }^{1}$ Three additional case reports ${ }^{2,3,5}$ and one small series ${ }^{4}$ have subsequently been reported. All reports describe patients with the adult-onset form of Still's disease. Prendiville et al. ${ }^{10}$ reported a series of juvenile patients with Still's-like symptoms, one of which was an 11-year old who displayed a persistent rash with clinical and pathological features similar to those described here. The lesions in this eruption are persistent (i.e. fixed or non-evanescent) and consist of pruritic papules and plaques with fine scale, most commonly located on the trunk, extremities, head and/or neck. In some cases the plaques display a 


\section{Fortna et al.}

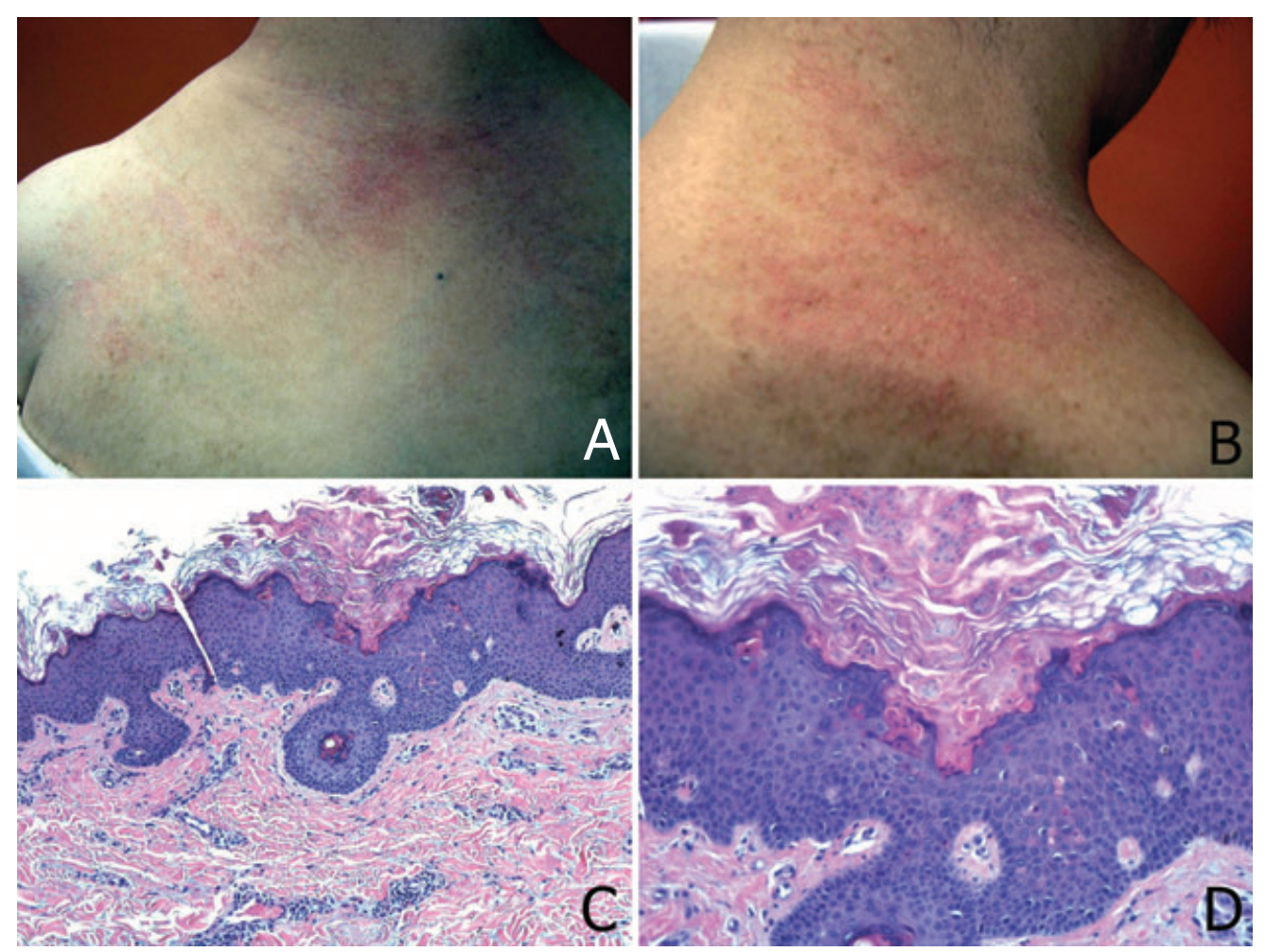

Fig. 3. Clinical and biopsy findings from patient 3. Persistent, erythematous, slightly scaly papules with somewhat linear configuration were present on the chest $(A)$ and upper back $(B)$. Medium $(C, \times 20)$ and high $(D, \times 40)$ power images of the biopsy show acanthosis and hyperkertosis with upper-layer dyskeratosis. A mild superficial perivascular infiltrate was present consisting of lymphocytes and scattered neutrophils. An increase in interstitial mucin was apparent in the dermis.

linear configuration, which has been suggested to represent a Koebner phenomenon. ${ }^{2,4,5}$ The histopathology of the eruption has two consistent findings: a characteristic pattern of dyskeratosis present mainly in the superficial layers of the epidermis (upper stratum spinosum, stratum granulosum and stratum corneum) without accompanying basilar dyskeratosis, and a sparse superficial dermal infiltrate often containing neutrophils but without vasculitis. The amount of dermal inflammation varies, and in some cases it has been minimal or absent. ${ }^{4,5}$ Most reported cases have also shown either orthokeratotic or parakeratotic hyperkeratosis. Although dyskeratosis in the lower epidermis is typically not seen, a few cases have shown focal vacuolar interface alterations and rare necrotic keratinocytes in the lower epidermis. ${ }^{4} \mathrm{Sev}$ eral other less specific findings have been reported in some patients, including dermal mucin deposition, ${ }^{5}$ subcorneal or intracorneal pustules, ${ }^{3}$ acanthosis and spongiosis. ${ }^{4}$ Evidence of external irritation (probably secondary to pruritus), such as excoriation or pigment incontinence, may be present in some cases. The histopathologic pattern of dyskeratosis with this clinical presentation is reminiscent of that seen in the verrucous phase of incontinentia pigmenti, although the two entities can easily be distinguished based on the clinical presentation.
While only recently recognized, 'persistent pruritic papules and plaques' of Still's disease appears to be present in a substantial subset of Still's patients. In the series of Lee et al., ${ }^{4} 65 \%$ (11/17) of their AOSD patients seen over a period of 17 years displayed this fixed rash, and biopsies performed on nine of those patients all had similar histopathologic features. The combined clinicopathologic features of this eruption are quite distinctive and, to our knowledge, not seen in any other condition. As the diagnosis of Still's disease is typically difficult and commonly delayed while other potential etiologies are excluded, recognition of these characteristic clinical and histopathologic patterns may significantly aid in the diagnosis of the condition.

Here, we have presented the clinical and histopathologic findings of three patients with the recently described fixed rash of Still's disease. Our findings confirm the two most salient histopathologic features of this eruption: dyskeratosis confined to the upper layers of the epidermis and a mild superficial dermal perivascular infiltrate containing neutrophils. One of our patients was a 15-year-old girl with a diagnosis of JIA, thus confirming that this eruption may be seen in either the adult or juvenile forms of the disease. 
Persistent pruritic papules and plaques in Still's disease

\section{Acknowledgement}

We are indebted to Dr. Diane Sun for assistance with the clinical photographs.

\section{References}

1. Lűbbe J, Hofer M, Chavaz P, Saurat JH, Borradori L. Adultonset Still's disease with persistent plaques. Br J Dermatol 1999; 141: 710 .

2. Suzuki K, Kimura Y, Aoki M, et al. Persistent plaques and linear pigmentation in adult-onset Still's disease. Dermatology 2001; 202: 333.

3. Affleck AG, Littlewood SM. Adult-onset Still's disease with atypical cutaneous features. J Eur Acad Dermatol Venereol 2005; 19: 360.

4. Lee JY-Y, Yang C-C, Hsu MM-L. Histopathology of persistent papules and plaques in adult-onset Still's disease. J Am Acad Dermatol 2005; 52: 1003.
5. Wolgamot G, YooJ, Hurst S, Gardner G, Olerud J, Argenyi Z. Unique histopathologic findings in a patient with adult-onset Still disease. Am J Dermatopathol 2007; 29: 194.

6. Fautrel B. Adult-onset Still disease. Best Pract Res Clin Rheumatol 2008; 22: 773.

7. Mehrpoor G, Owlia MB, Soleimani H, Mounzer K. Adultonset Still's disease: a report of 28 cases and review of the literature. Mod Rheumatol 2008; 18: 480.

8. Ravelli A, Martini A. Juvenile idiopathic arthritis. Lancet 2007; 369: 767.

9. Bywaters EG, Isdale IC. The rash of rheumatoid arthritis and Still's disease. QJ Med 1956; 25: 377.

10. Prendiville JS, Tucker LB, Cabral DA, et al. A pruritic linear urticarial rash, fever, and systemic inflammatory disease in five adolescents; adult-onset Still disease or systemic juvenile idiopathic arthritis sine arthritis? Pediatr Dermatol 2004; 21: 580 . 